\title{
Purple sweet potato color attenuates high fat-induced neuroinflammation in mouse brain by inhibiting MAPK and NF- $\kappa B$ activation
}

\author{
JIAN LI $^{1}, \mathrm{ZHAO} \mathrm{SHI}^{1}$ and YONGJIE MI ${ }^{1,2}$ \\ ${ }^{1}$ Department of Anatomy, Chengdu Medical College, Chengdu, Sichuan 610500; ${ }^{2}$ Department of Surgery, \\ Hebei University of Chinese Medicine, Shijiazhuang, Hebei 050200, P.R. China
}

Received May 10, 2016; Accepted March 30, 2017

DOI: $10.3892 / \mathrm{mmr} .2018 .8440$

\begin{abstract}
Purple sweet potato color (PSPC) is a natural anthocyanin pigment that is derived from purple sweet potato storage roots. PSPC possesses a variety of biological activities, including antioxidant, anti-inflammatory and neuroprotective effects; however, the detailed effects of PSPC on high-fat diet (HFD)-induced neuroinflammation remain to be determined. The aim of the present study was to investigate whether PSPC has a protective role in HFD-associated neuroinflammation in the mouse brain and to provide novel insight into the mechanisms of the action. C57BL $6 \mathrm{~J}$ mice were maintained on a normal diet (10 kcal\% fat), a HFD (60 kcal\% fat), a HFD with PSPC (700 mg/kg/day) or PSPC alone, which was administrated over 20 weeks. Open field and step-through tests were used to evaluate the effects of HFD and PSPC on mouse behavior and memory function. Western blotting and ELISA analyses were used to assess the expression of inflammatory cytokines and the activation of mitogen-activated protein kinase and nuclear factor- $\kappa \mathrm{B}(\mathrm{NF}-\kappa \mathrm{B})$. The results demonstrated that PSPC treatment was able to significantly improve the HFD-induced impairment of mouse behavior and memory function, and suppressed the increase in body weight, fat content, hyperlipemia and the level of endotoxin. PSPC treatment also markedly decreased the expression of cyclooxygenase- 2 , inducible nitric oxide synthase, tumor necrosis factor- $\alpha$, interleukin (IL)- $1 \beta$ and IL- 6 , and increased the level of IL-10 in the HFD-treated mouse brain. In addition,
\end{abstract}

Correspondence to: Professor Yongjie Mi, Department of Surgery, Hebei University of Chinese Medicine, 3 Xingyuan Road, Shijiazhuang, Hebei 050200, P.R. China

E-mail: yongjiemi@126.com

Abbreviations: BWG, body weight gain; ELISA, enzyme-linked immunosorbent assay; ERK, extracellular signal-regulated kinase; FC, fat content; HFD, high-fat diet; JNK, c-Jun N-terminal kinase; MAPK, mitogen-activated protein kinase; $N F-\kappa B$, nuclear factor- $\kappa \mathrm{B}$; PSPC, purple sweet potato color

Key words: neuroinflammation, high-fat diet, purple sweet potato color, mitogen-activated protein kinase, nuclear factor- $\mathrm{\kappa B}$
PSPC inhibited the HFD-induced phosphorylation of extracellular signal-regulated kinase (ERK), c-Jun N-terminal kinase (JNK) and p38, and the activation of NF- $\mathrm{NB}$. These findings indicated that PSPC treatment may alleviate HFD-induced neuroinflammation in the mouse brain by inhibiting ERK, JNK, p38 and NF- $\mathrm{B}$ activation.

\section{Introduction}

Obesity is a common trophic disorder that may be attributed to an imbalance of energy metabolism. Long-term, high-calorie food intake overwhelms energy expenditure, leading to excessive adipose accumulation in the body (1). Adipose tissue was originally considered a passive reservoir for energy storage, mechanical and heat insulation, and a regulator of thermogenesis (2); however, it is gradually becoming recognized that it can synthesize and secrete a number of bioactive peptides (known as adipokines) in an autocrine or paracrine manner (3). Adipokines serve a crucial role in regulating glucose and lipid metabolism, energy homeostasis, insulin sensitivity, immunity and inflammation (4). It is now widely accepted that obesity is highly associated with chronic, low-grade systemic inflammation in the body (5). A large body of evidence has indicated that high-fat diets (HFDs) are a major cause of metabolic disorders, such as obesity and obesity-associated diseases (6-8).

Over the past few decades, our diets have altered substantially, and in modern society the consumption of fatty foods has increased greatly. An epidemiological report revealed that a HFD causes hyperlipemia and is associated with detrimental effects on the brain (9). In addition, brain disorders induced by a HFD are usually accompanied by the onset of neuroinflammation, which is a key component in the progression of a number of neurological and neurodegenerative diseases (10). A recent study demonstrated that mice fed a HFD exhibited a greater susceptibility to kainic acid-induced oxidative stress and neuroinflammation in the hippocampus, and that neuroinflammation may contribute to neuronal death following brain injury (11). Reducing the overexpression of inflammatory cytokines in mouse brains prevents HFD-associated blood-brain barrier disruption and glial cell activation (12). Therefore, regulating the effects of neuroinflammation on the brain may be beneficial in decreasing the rates of neurodegenerative diseases, including 
Alzheimer's disease, Parkinson's disease and multiple sclerosis (13-15). However, there is a lack of effective treatments for obesity-associated neuroinflammation in the brain, which may have wide-ranging consequences to public health $(16,17)$. Therefore, the identification of effective preventative and therapeutic strategies are required.

A number of recent studies have focused on the pharmacological properties of natural products such as anthocyanins, which are a group of naturally occurring polyphenol compounds that are widely distributed in flowers, fruits and vegetables and are responsible for their intense color $(18,19)$. Anthocyanins have been reported to exhibit several potent biological properties, including anti-inflammation (20), anti-oxidation (21), anti-mutagen and anti-tumor (22). One natural anthocyanin pigment, purple sweet potato color (PSPC; which is derived from the storage roots of the purple sweet potato) has received increased attention owing to its unique color, and its nutritional and health-promoting benefits (23). High-performance liquid chromatography (HPLC) analysis has revealed that the main components of PSPC are acetylated cyanidin acyl glucosides and peonidin acyl glucosides ( $>90 \%)$, and the remaining components are other flavonoids (24). Compared with extracted pigments from other plants, PSPC is more stable and is directly absorbed and distributed in the blood (25). Therefore, it may be a suitable candidate for further development of physiologically functional food factors.

Previous studies have demonstrated that PSPC possesses various biological activities, including antioxidant, anti-inflammatory and neuroprotective effects (26-30). PSPC has been demonstrated to ameliorate d-galactose-mediated cognition deficits or liver injury by improving oxidative damage and inflammatory responses in the brain or liver of aging mice $(26,27)$. In addition, PSPC was reported to improve HFD-induced obesity in mice by reducing inflammation in the liver by blocking the adenosine monophosphate-activated protein kinase (AMPK) signaling pathway $(28,31)$ or by increasing the sensitivity of the liver to insulin by blocking nuclear factor (NF)- $\kappa \mathrm{B}$ signaling (29). Other recent studies have revealed that PSPC treatment improved HFD-induced kidney damage, d-galactose-induced premature endothelial senescence and endothelial dysfunction by inhibiting oxidative stress-mediated NACHT, LRR and PYD domains-containing protein 3 inflammasome activation in mice $(30,32)$. These results suggested that by investigating the protective mechanisms of PSPC treatment in the metabolic tissues associated with obesity, such as the brain, the development of treatments for obesity may be accelerated.

Multiple pharmacological effects of PSPC have been studied; however, the detailed effects of PSPC treatment on HFD-induced neuroinflammation have not yet been identified. The aim of the present study was to investigate whether PSPC treatment was able to improve spontaneous behavior, learning and memory functions in HFD-challenged mice. In addition, the present study aimed to determine whether PSPC has a protective role in HFD-associated neuroinflammation in the brain and to provide novel insight into the mechanisms of action.

\section{Materials and methods}

Reagents and drugs. PSPC (purity, >90\%) was purchased from Qingdao Pengyuan Natural Pigment Research Institute
(Qingdao, China). Unless otherwise noted, all reagents and drugs were purchased from Sigma-Aldrich; Merck KGaA (Darmstadt, Germany). Bioreagents for western blotting were purchased from Promega Corporation (Madison, WI, USA) or Cell Signaling Technology, Inc. (Danvers, MA, USA).

Animal procedures. A total of 60 male C57BL/6 mice (age, 8 weeks; weight, 20-24 g; Jackson Laboratory, Ben Harbor, ME, USA) were used in the present study. Mice were housed in a ventilated and temperature-controlled room (humidity, $60 \%$; temperature, $23 \pm 1^{\circ} \mathrm{C}$ ) with a $12 \mathrm{~h}$ light-dark cycle and free access to rodent chow and tap water; 3 mice were housed per cage. Following acclimation to the laboratory conditions, mice were randomly divided into the following 4 groups (15 mice/group): i) Control group, which were fed a normal diet containing $10 \mathrm{kcal} \%$ fat, $20 \mathrm{kcal} \%$ protein and 70 kcal\% carbohydrate (cat. no. D12450B; Research Diets, Inc., New Brunswick, NJ, USA); ii) HFD group, which were fed a HFD containing $60 \mathrm{kcal} \%$ fat, $20 \mathrm{kcal} \%$ protein and 20 kcal\% carbohydrate (cat. no. D12492; Research Diets, Inc.); iii) HFD + PSPC group, which were fed a HFD and were given PSPC (700 mg/kg body weight/day; in $0.9 \%$ normal saline) by oral gavage for 20 weeks, based as previously described $(21,23)$; and iv) PSPC group, which were fed a normal diet and were given the same daily dose of PSPC as the HFD + PSPC group. Following 20 weeks, mice were sacrificed and whole brain tissues were collected for experimental use or stored at $-80^{\circ} \mathrm{C}$. All animal care and experimental procedures were in accordance with the Chinese legislation on the use and care of laboratory animals, and experiments were approved by the Animal Supervision Committee of Chengdu Medical College (Sichuan, China). All studies involving animals are reported in accordance with the ARRIVE guidelines for reporting experiments involving animals (33).

\section{Behavioral tests}

Open field testing. Open field testing was performed as previously described (27), with minor modifications, to evaluate the effects of HFD and PSPC treatment on mouse behavior. Locomotor activity and exploratory behavior were examined in a circular arena (diameter, $50 \mathrm{~cm}$; height, $30 \mathrm{~cm}$ ) with a $40 \mathrm{~W}$ bulb (3,000 lux). The floor of the arena was divided into 21 equal units by white lines. Mice were allowed to acclimate for $1 \mathrm{~min}$ at the center of the arena prior to the experiment, the spontaneous behavioral variables were recorded within 5 min and were classified into 4 mutually exclusive categories, including: i) The distance mice travelled; ii) the speed mice travelled; iii) the number of rearing and leaning events; and iv) the number of grooming sessions.

Passive avoidance performance testing. Passive avoidance performance testing was performed as previously described (34), using an apparatus composed of two compartments, one illuminated and one dark, with a small hole in the wall separating the two. The illuminated compartment was lit with a $25 \mathrm{~W}$ bulb and the dark one contained a metal floor to conduct foot shocks $(36 \mathrm{~V} ; 50 \mathrm{~Hz})$. Each mouse was placed in the dimly lit room $3 \mathrm{~min}$ prior to the experiment to acclimate to the novel environment within the apparatus. Latency was defined as the interval between the initial entry into the hole and fully entering the dark compartment. Mice were placed individually 
into the illuminated compartment facing away from the hole in the wall. Once the mouse escaped to the dark compartment, a mild foot shock was administered for $5 \mathrm{sec}$ and the mouse was immediately removed from the apparatus. Following $24 \mathrm{~h}$, the mouse was placed in the illuminated compartment once more, following the aforementioned method, to carry out the retention test. The step-through latency within 5 min was recorded. The test was conducted on 2 consecutive days after the open-field testing.

Biochemical analysis. Body fat content (FC) was assessed using nuclear magnetic resonance-magnetic resonance imaging-based technology (EchoMRI LLC, Houston, TX, USA). At 20 weeks after the administration, blood samples were taken by tail venipuncture using heparin-coated capillary tube, and all rats were sacrificed by cervical dislocation without the use of an anesthetic. Blood samples were centrifuged at $1,000 \times \mathrm{g}$ for $10 \mathrm{~min}$ at $4^{\circ} \mathrm{C}$ to remove cell debris, and the serum was stored at $-20^{\circ} \mathrm{C}$ until measurements were taken. Serum levels of total cholesterol (TC), triglyceride (TG), high-density lipoprotein (HDL) and low-density lipoprotein (LDL) cholesterol were determined using the following reagent kits according to the manufacturer's protocol. TC assay kit (cat. no. A111-2), TG assay kit (cat. no. A110-2), HDL assay kit (cat. no. A112-2) and LDL assay kit (cat. no. A113-2), were obtained from Nanjing Jiancheng Bioengineering Institute (Nanjing, China). All experiments were performed in triplicate.

Enzyme-linked immunosorbent assay (ELISA). Following sacrifice, mice were perfused with $50 \mathrm{mM}$ chilled phosphate-buffered saline solution (PBS; $\mathrm{pH}$ 7.40). Mouse brains were harvested and the tissues were homogenized in PBS containing 0.1\% phenylmethylsulfonyl fluoride (Sigma-Aldrich; Merck KGaA). Following centrifugation at 16,000 x $\mathrm{g}$ for $15 \mathrm{~min}$ at $4^{\circ} \mathrm{C}$, the supernatant was collected. Quantitative measurements of the expression levels of inflammatory cytokines interleukin (IL)-6, IL-1 $\beta$, tumor necrosis factor (TNF)- $\alpha$ and IL-10 in mouse brains were performed with the following commercially ELISA kits according to the manufacturer's protocol: IL-6 (cat. no. R6000B), IL-1 $\beta$ (cat. no. RLB00), TNF- $\alpha$ (cat. no. RTA00), IL-10 (cat. no. R1000), obtained from R\&D Systems, Inc. (Minneapolis, MN, USA). ELISAs were conducted using an Infinite M200 PRO automated microplate reader (Tecan Group Ltd., Männedorf, Switzerland) at a wavelength of $540 \mathrm{~nm}$. All samples were tested in triplicate.

Western blot analysis. For western blot analysis, whole brain tissues were homogenized with CelLytic M lysis reagent and a protease inhibitor cocktail (Sigma-Aldrich; Merck $\mathrm{KGaA}$ ). Nuclear and cytoplasmic extracts for western blotting were obtained using the NE-PER Nuclear and Cytoplasmic Extraction reagents according to the manufacturer's protocol (Pierce; Thermo Fisher Scientific, Inc., Waltham, MA, USA). Protein concentrations were determined by the Bradford assay method. Samples were prepared with $5 \mathrm{X}$ loading buffer, then equal amounts $(40 \mu \mathrm{g})$ of total protein were separated by $10 \%$ SDS-PAGE and transferred to nitrocellulose membranes, which were rinsed with Tris-buffered saline containing $0.1 \%$ Tween-20 (TBS-T) and then blocked in TBS-T containing $5 \%$ nonfat dry milk for $1 \mathrm{~h}$ at room temperature. Membranes were incubated with the anti-iNOS rabbit mAb (cat. no. 13120; dilution, 1:1,000; Cell Signaling Technology, Inc., Danvers, MA, USA), anti-COX-2 rabbit mAb (cat. no. 12282; dilution, 1:1,000; Cell Signaling Technology, Inc.), anti-IL-6 rabbit mAb (cat. no. 12153; dilution, 1:1,000; Cell Signaling Technology, Inc.), anti-IL-1 $\beta$ rabbit $\mathrm{mAb}$ (cat. no. 12242; dilution, 1:1,000; Cell Signaling Technology, Inc.), anti-IL-10 rabbit mAb (cat. no. 12163; dilution, 1:1,000; Cell Signaling Technology, Inc.), anti-TNF- $\alpha$ rabbit mAb (cat. no. 11948; dilution, 1:1,000; Cell Signaling Technology, Inc.), anti- $\beta$-actin rabbit $\mathrm{mAb}$ (cat. no. 4970; dilution, 1:5,000; Cell Signaling Technology, Inc.) overnight at $4^{\circ} \mathrm{C}$, followed by washes in TBS-T and incubation with the horseradish peroxidase-conjugated secondary antibodies (cat. no. sc-2357; dilution, 1:10,000; Santa Cruz Biotechnology, Inc., Dallas, TX, USA) for $1 \mathrm{~h}$ at room temperature. Membranes were washed with TBS-T and bands were visualized using an enhanced chemiluminescence reagent (Invitrogen; Thermo Fisher Scientific, Inc.), according to the manufacturer's protocol. The intensity of the bands was analyzed using Quantity One software (version 4.6.2; Bio-Rad Laboratories, Inc., Hercules, CA, USA). The expression levels of all proteins in each sample were normalized to $\beta$-actin levels or K-70 protein, and the experiment was repeated three times.

Statistical analysis. Statistical analysis was performed using the Statistical Package for Social Sciences (SPSS, Inc., Chicago, IL, USA) software, version 16.0 for Windows. Data are presented as the mean \pm standard error of the mean. One-way analysis of variance followed by a Student-Newman-Keuls post hoc test, or an unpaired Student's t-test was used where appropriate for statistical analysis. $\mathrm{P}<0.05$ was considered to indicate a statistically significant difference.

\section{Results}

Effects of PSPC treatment on the general parameters of $H F D$-treated mice. A number of studies have demonstrated that a HFD is a risk factor for obesity or obesity-associated disease (6-8). In the present study, mice fed a HFD for 20 weeks exhibited a significant increase in body weight gain (BWG) and FC compared with mice in the Control group that were fed a normal diet (Table I). In addition, serum levels of TG, TC and LDL were significantly elevated, whereas serum levels of HDL were significantly decreased in HFD-treated mice compared with the Control group.

The HFD-induced increases in BWG, FC and serum TG, TC, LDL and LPS levels were all reduced, and serum HDL levels were increased in HFD + PSPC-treated mice, compared with mice in the HFD group. No significant differences were identified between the Control group and mice treated with PSPC alone and fed a normal diet.

\section{Effects of PSPC treatment on the behavior of HFD-treated mice}

Open field testing. To confirm whether locomotor activity and exploratory behavior were affected by HFD, an open field experiment was performed with mice following different treatments. As presented in Fig. 1, mice challenged with HDF for 20 weeks had a significant decrease in the distance and speed travelled (Fig. 1A and B), the number of grooming sessions 
Table I. Effects of purple sweet potato color on the different parameters in each group of mice.

\begin{tabular}{|c|c|c|c|c|}
\hline Parameter & Control & HFD & HFD + PSPC & PSPC \\
\hline Body weight gain (g) & $15.62 \pm 2.54$ & $28.23 \pm 3.81^{\mathrm{b}}$ & $19.78 \pm 3.66^{c}$ & $14.83 \pm 3.04$ \\
\hline $\mathrm{FC}^{\mathrm{a}}$ (\% of body weight) & $9.78 \pm 1.33$ & $20.45 \pm 1.98^{\mathrm{b}}$ & $13.57 \pm 1.19^{\mathrm{d}}$ & $10.15 \pm 1.28$ \\
\hline Serum TC (mmol/l) & $2.98 \pm 0.48$ & $3.84 \pm 0.37^{\mathrm{b}}$ & $3.21 \pm 0.24^{\mathrm{d}}$ & $3.15 \pm 0.33$ \\
\hline Serum TG (mmol/l) & $1.04 \pm 0.15$ & $1.75 \pm 0.25^{\mathrm{b}}$ & $1.29 \pm 0.18^{\mathrm{d}}$ & $1.20 \pm 0.17$ \\
\hline Serum HDL (mmol/l) & $1.58 \pm 0.36$ & $0.96 \pm 0.28^{b}$ & $1.34 \pm 0.42^{c}$ & $1.46 \pm 0.45$ \\
\hline Serum LDL (mmol/l) & $1.37 \pm 0.26$ & $2.67 \pm 0.42^{b}$ & $1.75 \pm 0.28^{\mathrm{d}}$ & $1.58 \pm 0.37$ \\
\hline
\end{tabular}

Data are presented as the mean \pm standard error of the mean ( $n=15 /$ group). ${ }^{a}$ Nuclear magnetic resonance-magnetic resonance imaging-based technology was used to assess body fat content and biochemical analysis of mouse blood samples was performed to investigate the other parameters. ${ }^{b} \mathrm{P}<0.01$ vs. Control group; ${ }^{\mathrm{C}} \mathrm{P}<0.05$ and ${ }^{\mathrm{d}} \mathrm{P}<0.01$ vs. HFD group. FC, fat content; HDL, high-density lipoprotein; HFD, high-fat diet; LDL, low-density lipoprotein; PSPC, purple sweet potato color; TC, total cholesterol; TG, triglyceride.
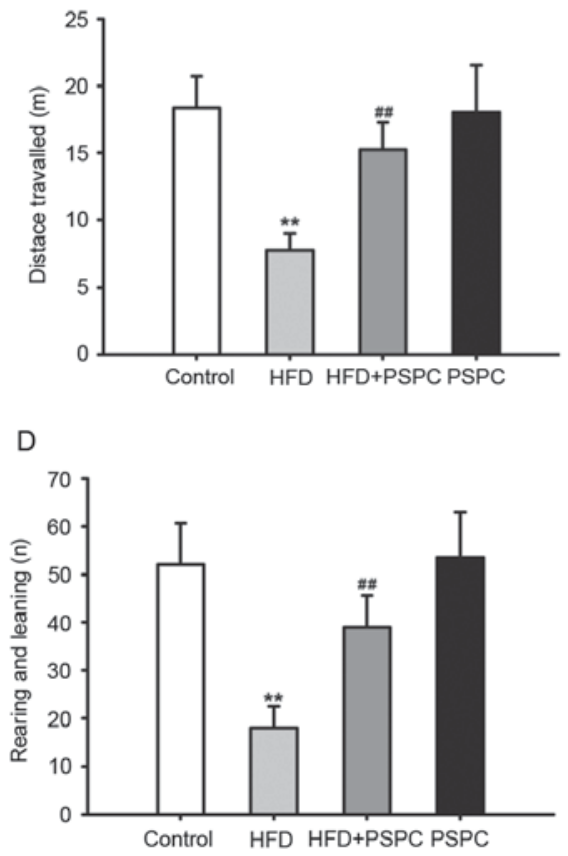

B

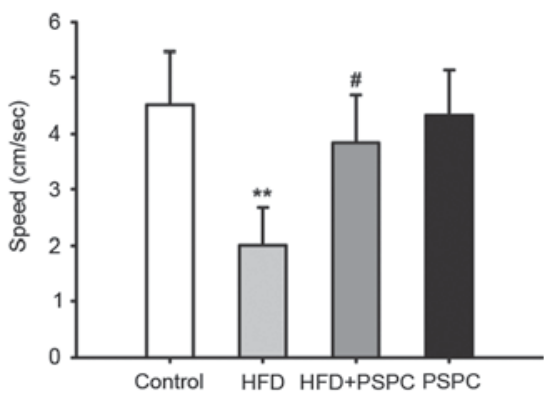

E

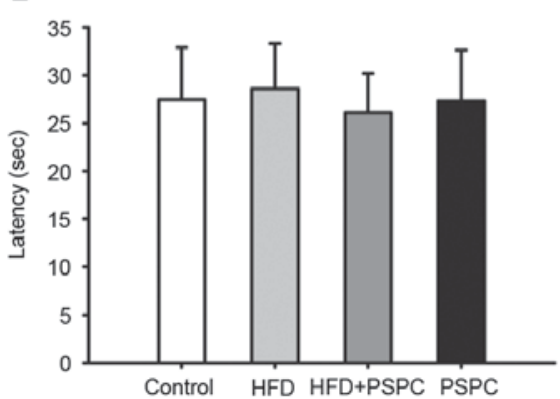

C

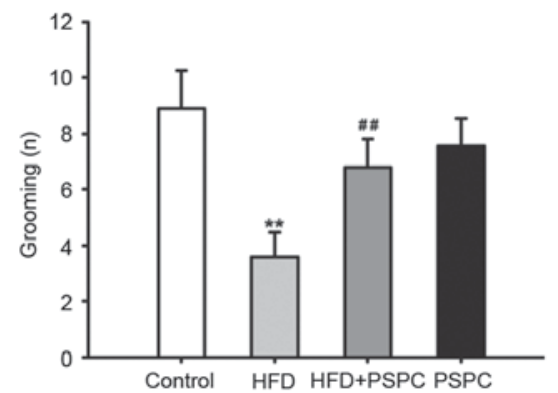

$\mathrm{F}$

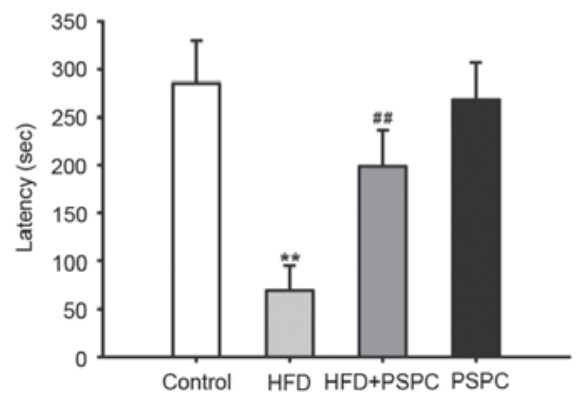

Figure 1. Effects of PSPC treatment on spontaneous behavior and memory function in HFD-treated mouse (n=15). Mice in each experimental group were evaluated for (A) distance and (B) speed travelled, (C) the number of rearing and leaning events, and (D) the number of grooming sessions. Latency in the (E) initial trial and (F) 24-h retention trial of each group in the step-through test. Data are presented as the mean \pm standard error of the mean. ${ }^{* * *} \mathrm{P}<0.01$ vs. Control group; ${ }^{\#} \mathrm{P}<0.05$ and ${ }^{\# \#} \mathrm{P}<0.01$ vs. HFD group. HFD, high fat diet; $\mathrm{PSPC}$, purple sweet potato color.

(Fig. 1C) and the number of rearing and leaning events (Fig. 1D) compared with mice in the Control group, indicating that mice challenged with HFD had impaired locomotor activity and exploratory behavior. When HFD-treated mice were co-treated with PSPC the impaired activities and behaviors significantly improved (Fig. 1A-D). There were no significant differences identified between the Control and PSPC-only groups for all parameters measured.

Passive avoidance performance testing. In the initial part of the step-through test, no significant differences were identified between the latencies among the four groups (Fig. 1E). However, following a 5 sec mild foot shock in the dark compartment, the latency in the 24-h retention test was significantly decreased in HFD-treated mice compared with the Control group. By contrast, PSPC treatment markedly increased the latency in HFD + PSPC mice compared with mice only fed a HFD (Fig. 1F). These results suggest that PSPC may be able to improve the deficits in memory function of mice to a certain degree. However, no significant differences were identified between the mice challenged with PSPC alone and the Control group.

Effects of PSPC treatment on cyclooxygenase-2 (COX-2) and inducible nitric oxide synthase (iNOS) protein expression levels in HFD-treated mouse brains. COX-2 and iNOS are important regulators in the progression of inflammation, whose expression levels are relatively low in normal tissues (35). However, when stimulated with endotoxin or inflammatory cytokines, such as IL-6, IL-1 $\beta$ or TNF- $\alpha$, their expression levels markedly increase. In the present study 
western blot analysis was performed to determine the effect of a HFD and PSPC treatment on the expression of COX-2 and iNOS proteins. HFD treatment significantly upregulated the expression levels of COX-2 and iNOS compared with Control mice; this elevated expression was significantly reduced in HFD-fed mice co-treated with PSPC (Fig. 2A and B). No significant differences were identified between the Control and PSPC groups.

Effects of PSPC treatment on the expression levels of neuroinflammatory cytokines in HFD-treated mice. Neuroinflammatory cytokines are important contributors to the pathogenesis and development of cognitive impairment, and increased levels of expression have been reported to disrupt hippocampal synaptic plasticity (36). Therefore, the effects of a HFD and PSPC treatment on the levels of neuroinflammatory cytokines, including TNF- $\alpha$, IL-6, IL-1 $\beta$ and IL-10 in the mouse brain were determined by western blotting and ELISA. As shown in Fig. 3A and B, mice fed a HFD exhibited a significant increase in the protein expression levels of the proinflammatory cytokines IL-6, IL-1 $\beta$ and TNF- $\alpha$, and a decrease in the expression of anti-inflammatory cytokine IL-10, compared with Control mice. However, these effects were significantly reversed by PSPC treatment in the HFD + PSPC group compared with the HFD group. Results from ELISA analysis confirmed the effects of HFD and PSPC treatment on the production of neuroinflammatory cytokines in the mouse brain (Fig. 3C). A significant increase in the expression levels of IL- 6 , IL- $1 \beta$ and TNF- $\alpha$ was observed, in addition to a marked decrease in the level of IL-10 in the brain of HFD-fed mice, compared with the Control group. PSPC markedly decreased the expression levels of IL- 6 by $43.93 \%$, IL- $1 \beta$ by $32.25 \%$ and TNF- $\alpha$ by $42.42 \%$, and increased the level of IL-10 by $40.82 \%$ in the HFD + PSPC group compared with the HFD-only group (Fig. 3C). No significant differences were identified between the PSPC group and the Control group in either the western blotting or ELISA analyses.

Effects of PSPC treatment on HFD-induced mitogen-activated protein kinase $(M A P K)$ and $N F-\kappa B$ activation in mouse brains. A number of previous studies have demonstrated that MAPK and $\mathrm{NF}-\kappa \mathrm{B}$ activation serves a crucial role in the progression of inflammation $(37,38)$. To further determine the molecular mechanisms underlying the suppressive effects of PSPC on neuroinflammation in the mouse brain, the effects of PSPC on the expression and phosphorylation levels of the MAPK family members extracellular signal-regulated kinase (ERK), c-Jun N-terminal kinase (JNK), and p38 were determined by western blot analysis. The brains of mice fed a HFD had significantly increased expression levels of phosphorylated (p)-ERK, p-JNK and p-p38 proteins compared with Control mice (Fig. 4A and B). However, this increase in p-ERK, p-JNK and p-p38 protein expression levels in mice challenged with a HFD was significantly reduced by PSPC treatment (Fig. 4A and B).

$\mathrm{NF}-\kappa \mathrm{B}$ activation induces its translocation from the cytoplasm to the nucleus. The present study investigated the nuclear and cytoplasmic expression levels of NF- $\mathrm{B}$ p65 (Fig. 4C and D). NF- $\kappa$ B p65 levels in the nuclear fractions were significantly increased in HFD-treated mice compared

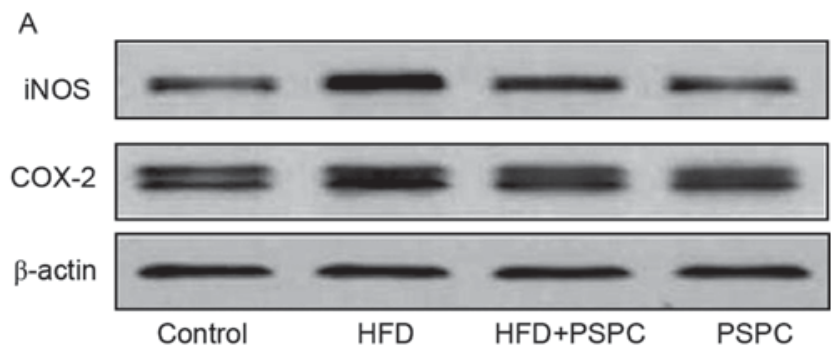

B

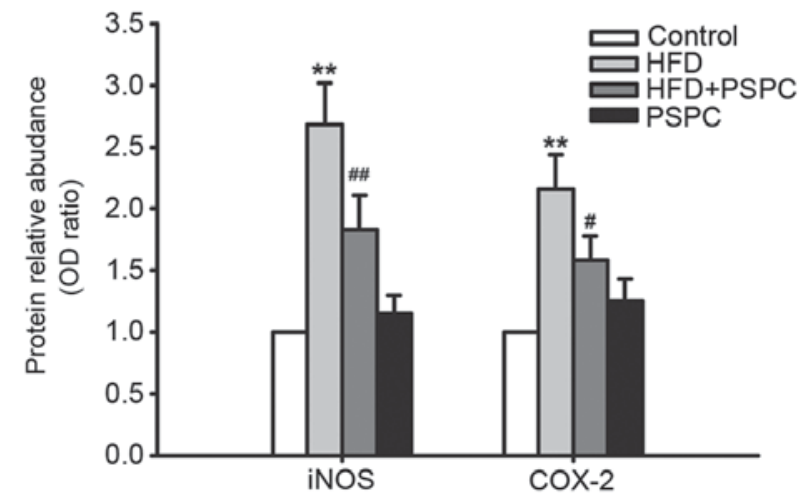

Figure 2. Effects of PSPC treatment on COX-2 and iNOS protein expression levels in HFD-treated mouse brains $(n=5)$. (A) Western blots demonstrating the expression levels of iNOS and COX-2 in brain tissues from mice in each of the indicated experimental conditions. (B) COX-2 and iNOS expression levels were normalized to $\beta$-actin; expression levels in the Control group were set to 1.0. Data are presented as the mean \pm standard error of the mean. ${ }^{* *} \mathrm{P}<0.01$ vs. Control group; ${ }^{\#} \mathrm{P}<0.05$ and ${ }^{\# \#} \mathrm{P}<0.01$ vs. HFD group. COX-2, cyclooxygenase-2; HFD, high fat diet; iNOS, inducible nitric oxide synthase; PSPC, purple sweet potato color.

with Control mice, whereas the cytoplasmic expression levels were significantly decreased in HFD-treated mice. PSPC treatment in mice fed a HFD inhibited the activation of $\mathrm{NF}-\kappa \mathrm{B}$, as demonstrated by the increased cytoplasmic expression of $\mathrm{NF}-\kappa \mathrm{B}$ p65 and the decreased levels in the nuclear fractions comparing the HFD-only group. No statistically significant differences were identified between the PSPC and Control groups for MAPK phosphorylation or $\mathrm{NF}-\kappa \mathrm{B}$ activation.

\section{Discussion}

An increasing number of studies have demonstrated that a HFD contributed to the development of obesity and fat deposition in a variety of tissues and is associated with hyperlipemia and detrimental effects on the brain (9,11). HFD-induced chronic inflammation, primarily elicited by glial cells in the brain, serves a central role in cognition and memory impairment, as well as in neurodegenerative diseases $(10,39)$. Neuroinflammation is a complex process that involves a diverse set of proinflammatory cytokines and reactive oxygen species (ROS), which may be detrimental to neuronal health and may reduce synaptic plasticity $(12,36)$. Therefore, novel therapeutic strategies are required that will improve obesity-associated metabolic perturbations and neuroinflammation. PSPC belongs to a class of naturally occurring anthocyanins, which are used to give food color, and has recently been reported to have a number of pharmacological activities, including as potent antioxidant, anti-inflammatory and neuroprotective 
A

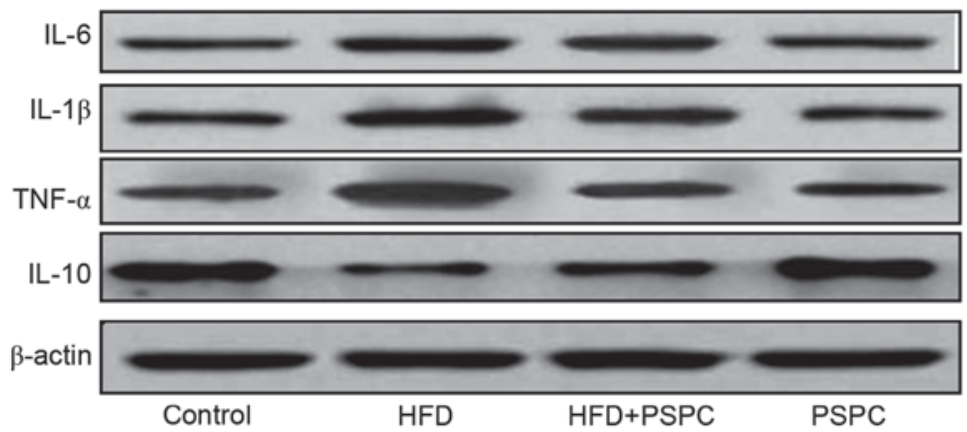

B

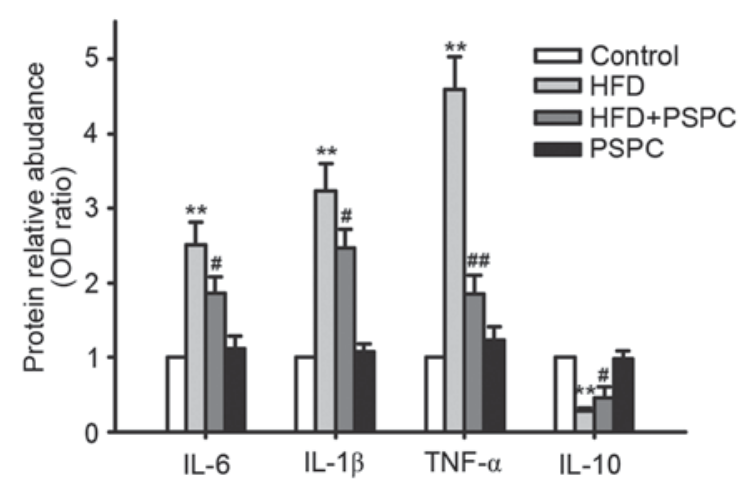

C

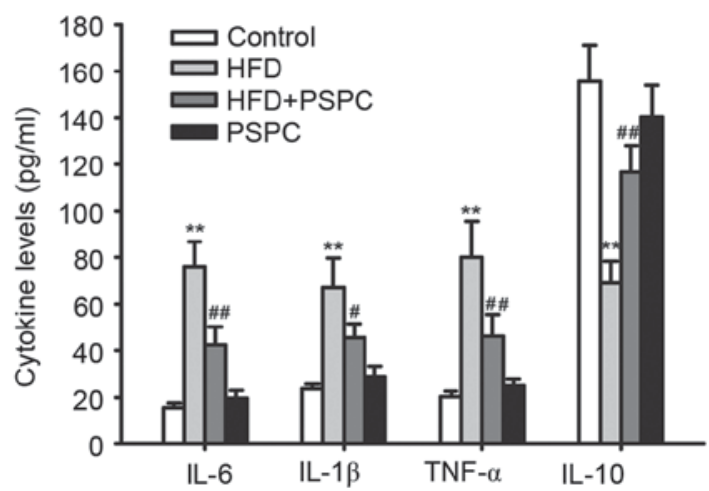

Figure 3. Effects of PSPC treatment on the expression of neuroinflammatory cytokines in HFD-treated mouse brains (n=5). (A) Western blots revealing the expression levels of the proinflammatory cytokines IL-6, IL-1 $\beta$ and TNF- $\alpha$ and the anti-inflammatory cytokine IL-10 in the brain tissues of mice from each group under the indicated experimental conditions. (B) Expression levels of the neuroinflammatory cytokines were normalized to $\beta$-actin; expression levels in the Control group were set to 1.0. (C) ELISA analysis of the levels of neuroinflammatory cytokines produced in mouse brains. Data are presented as the mean \pm standard error of the mean. ${ }^{* *} \mathrm{P}<0.01$ vs. Control group; ${ }^{\#} \mathrm{P}<0.05$ and ${ }^{\# \#} \mathrm{P}<0.01$ vs. HFD group. HFD, high fat diet; IL, interleukin; PSPC, purple sweet potato color; TNF- $\alpha$, tumor necrosis factor- $\alpha$.

effects (26-30). In the present study, the effects of PSPC on HFD-induced neuroinflammation in the mouse brain was investigated following 20 weeks of daily administration of PSPC (700 mg/kg body weight/day) via oral gavage.

The results of biochemical analysis revealed that HFD treatment induced hyperlipemia and significantly increased BWG and $\mathrm{FC}$ in mice, which was consistent with a previous report (11). However, the HFD-induced increases in BWG, FC and hyperlipemia were all suppressed by PSPC co-treatment. In behavioral tests, HFD-treatment resulted in a marked impairment of mouse behavior and memory function. HFD + PSPC-treated mice exhibited improved active behavioral responses to the open field test, and PSPC treatment inhibited HFD-induced behavioral retrogression, including distance and speed travelled, and the number of grooming and rearing and leaning events. In addition, PSPC treatment also restored the HFD-induced memory impairment to some extent in the step-through tasks.

To characterize the effects of PSPC on neuroinflammation in the mouse brain, the expression profile of two mediators of inflammation, iNOS and COX-2, were detected in mouse brains. Previous studies have implicated iNOS expression in neuronal damage and death in a number of central nervous system-associated diseases (40), and COX-2 expression was reported to be induced by inflammatory stimuli in the hippocampus (41). In the present study, western blot analyses revealed that HFD treatment upregulated the expression levels of iNOS and COX-2 proteins, which were significantly reduced following PSPC administration. In addition, the expression levels of neuroinflammatory cytokines IL-6, IL-1 $\beta$, IL-10 and TNF- $\alpha$ were determined in mouse brains by western blotting and ELISA analyses, as these cytokines have a crucial role in the progression of inflammation. Western blotting and ELISA results demonstrated that the expression levels of IL-6, IL-1 $\beta$ and TNF- $\alpha$ were elevated, and the level of IL-10 was decreased in mice fed a HFD, which was comparable to the results of a study in diet-induced obese animal models (36). However, these alterations in the levels of neuroinflammatory cytokines were attenuated by PSPC treatment, confirming the anti-inflammatory and neuroprotective effects of PSPC.

To further determine the mechanisms underlying the PSPC-induced suppression of neuroinflammation in mouse brains, the present study investigated the effects of PSPC on the expression levels of MAPK family members ERK, JNK and p38 proteins, as MAPK activation is known to be involved in the expression of proinflammatory cytokines (42). Activated ERK has been demonstrated to have a neurotoxic effect, JNK was revealed to be involved in cell apoptotic responses and p38 was reported to participate in neuronal responses to various stressors (43). The results of the present study revealed that PSPC treatment ameliorated the HFD-induced phosphorylation/activation of ERK, JNK and p38 MAPKs in mouse brains. These results are comparable to those from a previous study that demonstrated that ERK and JNK are involved in the LPS-induced acute inflammatory response in mouse brain (44). However, they did not detect a significant difference in p38 expression with LPS-treatment mice, which 
A

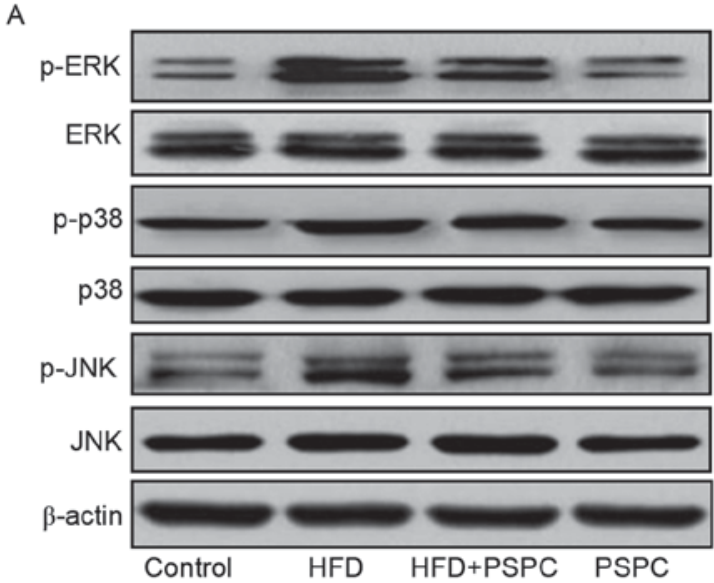

C
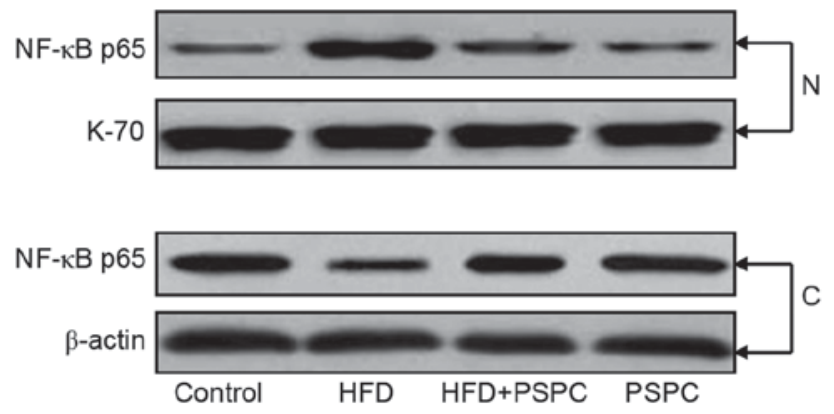

B

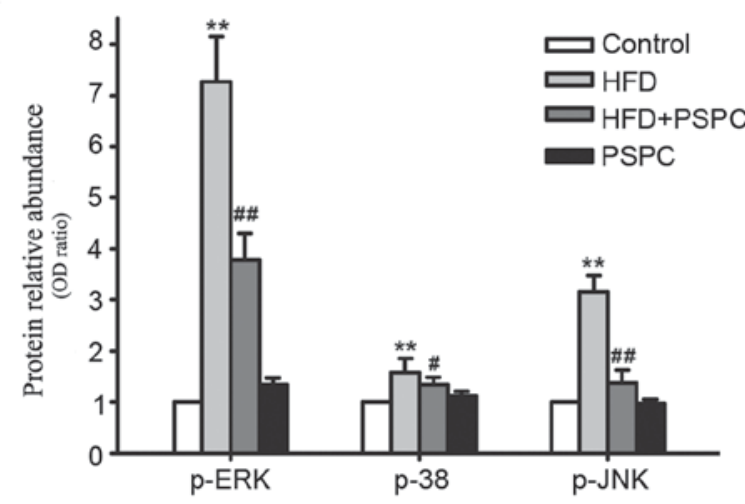

D

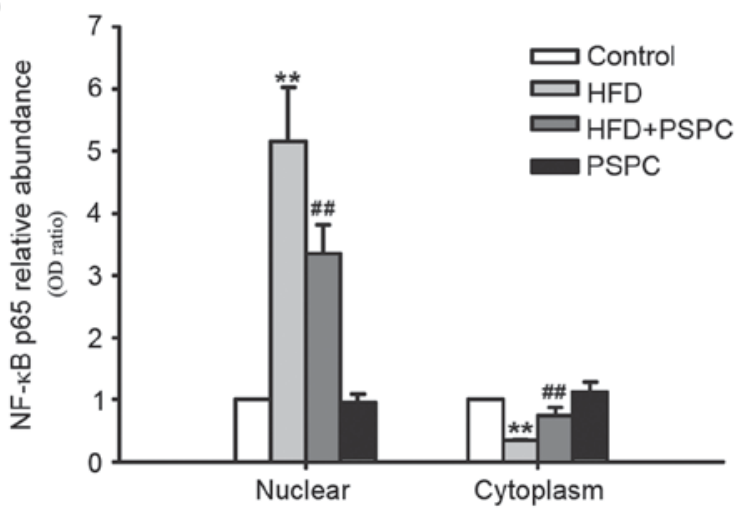

Figure 4. Effects of PSPC treatment on HFD-induced MAPK phosphorylation and NF-kB activation in mouse brains (n=5). (A) Western blots revealing the expression levels of p-ERK, ERK, p-p38, p38, p-JNK and JNK. (B) Densitometric analysis of the proteins in (A); expression levels were normalized to $\beta$-actin and expressions in the Control group were set to 1.0. (C) Western blots revealing the expression levels of NF- $\mathrm{kB}$ p65 protein in nuclear and cytoplasmic fractions in the brain tissues of mice from each group under the indicated experimental conditions. (D) Densitometric analysis of the proteins in (C); nuclear and cytoplasmic expression levels were normalized to $\beta$-actin or K-70, respectively; expressions in the Control group were set to 1.0. Data are presented as the mean \pm standard error of the mean. ${ }^{* *} \mathrm{P}<0.01$ vs. Control group; ${ }^{*} \mathrm{P}<0.05$ and ${ }^{\# \prime} \mathrm{P}<0.01$ vs. HFD group. $\mathrm{C}$, cytoplasm; ERK, extracellular signal-regulated kinase; HFD, high fat diet; JNK, c-Jun N-terminal kinase; MAPK, mitogen-activated protein kinase; N, nuclear; NF-кB, nuclear factor-kB; p-, phosphorylated; PSPC, purple sweet potato color.

is not consistent with the results of the present study. Based on these results, the MAPK pathway may be involved in HFD-induced neuroinflammation and PSPC treatment may exert an anti-inflammatory effect on the mouse brain, at least in part, by inhibiting the phosphorylation of ERK, JNK and p38 MAPKs.

$\mathrm{NF}-\kappa \mathrm{B}$ is a transcription regulator that serves important roles in cell proliferation and survival, inflammation and immunity, and is considered the main signaling pathway that is involved in the development of inflammatory diseases, including hepatitis, nerve injury and lung injury (45). NF- $\mathrm{B}$ is composed of two subunits, p50 and p65, which form a heterodimer that is bound by members of the inhibitors of $\kappa \mathrm{B}(\mathrm{I} \kappa \mathrm{B})$ family of inhibitor proteins and is sequestered in the cytoplasm under resting conditions. When exposed to various stimuli that activate $\mathrm{NF}-\kappa \mathrm{B}, \mathrm{I} \kappa \mathrm{B}$ proteins are phosphorylated and subsequently ubiquitinated and degraded. The unbound NF- $\kappa \mathrm{B}$ dimers translocate to the nucleus where they bind to cis-regulatory elements called $\mathrm{kB}$ sites in target genes and initiate transcription (46). In the nervous system, $\mathrm{NF}-\kappa \mathrm{B}$ is activated by a number of stimuli, including ROS, neurotrophic factors and neurotransmitters, and has been previously reported to function in the control of cell growth, cell survival, inflammatory response, synaptic plasticity, behavior and cognition (37). Considering the potential role of $\mathrm{NF}-\kappa \mathrm{B}$ in neuroinflammation, the present study examined the activation of $\mathrm{NF}-\kappa \mathrm{B}$ in the brain tissues of mice challenged with HFD and treated with PSPC. The results indicated that a HFD induced a significant decrease in the cytoplasmic expression of $\mathrm{NF}-\kappa \mathrm{B}$ subunit $\mathrm{p} 65$ and a significant increase in nuclear expression, suggesting that HFD may promote NF- $\kappa \mathrm{B}$ activation in mouse brains. This effect was inhibited when mice were treated with PSPC, which is consistent with the levels of LPS detected in the serum. LPS is a Gram-negative bacterial endotoxin, which activates the biosynthesis of proinflammatory cytokines by binding to toll-like receptor 4 and inducing signaling by activating NF- $\mathrm{B}$ (47), suggesting that PSPC may exert anti-neuroinflammatory actions in the mouse brain by decreasing the levels of LPS and the subsequent inhibition of $\mathrm{NF}-\kappa \mathrm{B}$ activation.

To clarify whether PSPC was able to affect mouse metabolism and physiological processes, the present study compared the aforementioned characteristics between the experimental group receiving PSPC alone and the Control group; both were fed a normal diet. The results demonstrated that there were no significant differences in the biochemical parameters or in 
the expression levels of iNOS, COX-2 and the inflammatory cytokines, or in the phosphorylation/activation of MAPKs and $\mathrm{NF}-\kappa \mathrm{B}$, indicating that PSPC itself may not alter metabolism and physiological processes in mice. These results further confirmed that the observed behavior and biochemical alterations may be due to the ameliorating effect of PSPC on HFD-induced neuroinflammation in mouse brains. The present study confirmed the anti-inflammatory and neuroprotective effects of PSPC.

In conclusion, the present study demonstrated that PSPC attenuates HFD-induced neuroinflammation, and thereby improves behavior and memory function impairments in mouse brains by downregulating the expression of iNOS, COX-2, IL-1 $\beta$, IL- 6 and TNF- $\alpha$, and upregulating the expression of IL-10, through the suppression of ERK, JNK and p38 MAPK phosphorylation and $\mathrm{NF}-\kappa \mathrm{B}$ signaling pathway activation. These findings about the pharmacological efficacy of PSPC may offer a novel therapeutic approach to ameliorate obesity-associated neuroinflammation.

\section{Acknowledgements}

The present study was funded by a Special Grant from the Key Laboratory of Sichuan Province for Developmental Regeneration (grant no. SYS10-006).

\section{References}

1. Meissburger B, Ukropec J, Roeder E, Beaton N, Geiger M, Teupser D, Civan B, Langhans W, Nawroth PP, Gasperikova D, et al: Adipogenesis and insulin sensitivity in obesity are regulated by retinoid-related orphan receptor gamma. EMBO Mol Med 3: 637-651, 2011

2. Trayhurn P and Beattie JH: Physiological role of adipose tissue: White adipose tissue as an endocrine and secretory organ. Proc Nutr Soc 60: 329-339, 2001.

3. Proença AR,SertiéRA, Oliveira AC,Campaña AB,CaminhottoRO, Chimin P and Lima FB: New concepts in white adipose tissue physiology. Braz J Med Biol Res 47: 192-205, 2014.

4. Smitka K and Marešová D: Adipose tissue as an endocrine organ: An update on pro-inflammatory and anti-inflammatory microenvironment. Prague Med Rep 116: 87-111, 2015.

5. Wellen KE and Hotamisligil GS: Obesity-induced inflammatory changes in adipose tissue. J Clin Invest 112: 1785-1788, 2003.

6. Golay A and Bobbioni E: The role of dietary fat in obesity. Int J Obes Relat Metab Disord 21 (Suppl 3): S2-S11, 1997.

7. Ginsberg HN, Zhang YL and Hernandez-Ono A: Metabolic syndrome: Focus on dyslipidemia. Obesity (Silver Spring) 14 (Suppl 1): 41S-49S, 2006.

8. Seidell JC: Dietary fat and obesity: An epidemiologic perspective. Am J Clin Nutr 67 (3 Suppl): 546S-550S, 1998

9. Freeman LR, Haley-Zitlin V, Stevens C and Granholm AC: Diet-induced effects on neuronal and glial elements in the middle-aged rat hippocampus. Nutr Neurosci 14: 32-44, 2011.

10. Thirumangalakudi L, Prakasam A, Zhang R, Bimonte-Nelson $\mathrm{H}$, Sambamurti K, Kindy MS and Bhat NR: High cholesterol-induced neuroinflammation and amyloid precursor protein processing correlate with loss of working memory in mice. J Neurochem 106: 475-485, 2008

11. Kang DH, Heo RW, Yi CO, Kim H, Choi $\mathrm{CH}$ and Roh GS: High-fat diet-induced obesity exacerbates kainic acid-induced hippocampal cell death. BMC Neurosci 16: 72, 2015.

12. Nerurkar PV, Johns LM, Buesa LM, Kipyakwai G, Volper E, Sato R, Shah P, Feher D, Williams PG and Nerurkar VR: Momordica charantia (bitter melon) attenuates high-fat diet-associated oxidative stress and neuroinflammation. J Neuroinflammation 8: 64, 2011.

13. Orr CF, Rowe DB and Halliday GM: An inflammatory review of Parkinson's disease. Prog Neurobiol 68: 325-340, 2002.

14. Qian L, Flood PM and Hong JS: Neuroinflammation is a key player in Parkinson's disease and a prime target for therapy. J Neural Transm (Vienna) 117: 971-979, 2010.
15. Lee YJ, Han SB, Nam SY, Oh KW and Hong JT: Inflammation and Alzhe-imer's disease. Arch Pharm Res 33: 1539-1556, 2010.

16. Simmons RK, Alberti KG, Gale EA, Colagiuri S, Tuomilehto J, Qiao Q, Ramachandran A, Tajima N, Brajkovich Mirchov I, Ben-Nakhi A, et al: The metabolic syndrome: Useful concept or clinical tool? Report of a WHO Expert Consultation. Diabetologia 53: 600-605, 2010.

17. Palacios N, Gao X, McCullough ML, Jacobs EJ, Patel AV, Mayo T, Schwarzschild MA and Ascherio A: Obesity, diabetes, and risk of Parkinson's disease. Mov Disord 26: 2253-2259, 2011.

18. Iwashina T: Contribution to flower colors of flavonoids including anthocyanins: A review. Nat Prod Commun 10: 529-544, 2015.

19. He J and Giusti MM: Anthocyanins: Natural colorants with health-promoting properties. Annu Rev Food Sci Technol 1: 163-187, 2010.

20. Huang WY, Liu YM, Wang J, Wang XN and Li CY: Anti-inflammatory effect of the blueberry anthocyanins malvidin-3-glucoside and malvidin-3-galactoside in endothelial cells. Molecules 19: 12827-12841, 2014.

21. Pool-Zobel BL, Bub A, Schröder N and Rechkemmer G: Anthocyanins are potent antioxidants in model systems but do not reduce endogenous oxidative DNA damage in human colon cells. Eur J Nutr 38: 227-234, 1999.

22. Kang HG, Jeong SH and Cho JH: Antimutagenic and anticarcinogenic effect of methanol extracts of sweetpotato (Ipomea batata) leaves. Toxicol Res 26: 29-35, 2010.

23. Mano H, Ogasawara F, Sato K, Higo H and Minobe Y: Isolation of a regulatory gene of anthocyanin biosynthesis in tuberous roots of purple-fleshed sweet potato. Plant Physiol 143: 1252-1268, 2007.

24. Qiu F, Luo J, Yao S, Ma L and Kong L: Preparative isolation and purification of anthocyanins from purple sweet potato by high-speed counter-current chromatography. J Sep Sci 32: 2146-2151, 2009.

25. Harada K, Kano M, Takayanagi T, Yamakawa O and Ishikawa F: Absorption of acylated anthocyanins in rats and humans after ingesting an extract of Ipomoea batatas purple sweet potato tuber. Biosci Biotechnol Biochem 68: 1500-1507, 2004.

26. Zhang ZF, Fan SH, Zheng YL, Lu J, Wu DM, Shan Q and Hu B: Purple sweet potato color attenuates oxidative stress and inflammatory response induced by d-galactose in mouse liver. Food Chem Toxicol 47: 496-501, 2009.

27. Shan Q, Lu J, Zheng Y, Li J, Zhou Z, Hu B, Zhang Z, Fan S, Mao Z, Wang YJ and Ma D: Purple sweet potato color ameliorates cognition deficits and attenuates oxidative damage and inflammation in aging mouse brain induced by d-galactose. J Biomed Biotechnol 2009: 564737, 2009.

28. Hwang YP, Choi JH, Yun HJ, Han EH, Kim HG, Kim JY, Park BH, Khanal T, Choi JM, Chung YC and Jeong HG: Anthocyanins from purple sweet potato attenuate dimethylnitrosamine-induced liver injury in rats by inducing Nrf2-mediated antioxidant enzymes and reducing COX-2 and iNOS expression. Food Chem Toxicol 49: 93-99, 2011.

29. Zhang ZF, Lu J, Zheng YL, Wu DM, Hu B, Shan Q, Cheng W, Li MQ and Sun YY: Purple sweet potato color attenuates hepatic insulin resistance via blocking oxidative stress and endoplasmic reticulum stress in high-fat-diet-treated mice. J Nutr Biochem 24: 1008-1018, 2013.

30. Shan Q, Zheng Y, Lu J, Zhang Z, Wu D, Fan S, Hu B, Cai X, Cai H, Liu P and Liu F: Purple sweet potato color ameliorates kidney damage via inhibiting oxidative stress mediated NLRP3 inflammasome activation in high fat diet mice. Food Chem Toxicol 69: 339-346, 2014.

31. Hwang YP, Choi JH, Han EH, Kim HG, Wee JH, Jung KO, Jung KH, Kwon KI, Jeong TC, Chung YC and Jeong HG: Purple sweet potato anthocyanins attenuate hepatic lipid accumulation through activating adenosine monophosphate-activated protein kinase in human HepG2 cells and obese mice. Nutr Res 31: 896-906, 2011.

32. Sun C, Fan S, Wang X, Lu J, Zhang Z, Wu D, Shan Q and Zheng Y: Purple sweet potato color inhibits endothelial premature senescence by blocking the NLRP3 inflammasome. J Nutr Biochem 26: 1029-1040, 2015.

33. McGrath JC, Drummond GB, McLachlan EM, Kilkenny C and Wainwright CL: Guidelines for reporting experiments involving animals: The ARRIVE guidelines. Br J Pharmacol 160: 1573-1576, 2010

34. Castellano C, Introini-Collison IB and McGaugh JL: Interaction of beta-endorphin and GABAergic drugs in the regulation of memory storage. Behav Neural Biol 60: 123-128, 1993. 
35. Jain NK, Ishikawa TO, Spigelman I and Herschman HR: COX-2 expression and function in the hyperalgesic response to paw inflammation in mice. Prostaglandins Leukot Essent Fatty Acids 79: 183-190, 2008.

36. Wang D, Liu L, Yan J, Wu W, Zhu X and Wang Y: Cardiotrophin-1 (CT-1) improves high fat diet-induced cognitive deficits in mice. Neurochem Res 40: 843-853, 2015.

37. Mémet S: NF-kappaB functions in the nervous system: From development to disease. Biochem Pharmacol 72: 1180-1195, 2006.

38. Arthur JS and Ley SC: Mitogen-activated protein kinases in innate immunity. Nat Rev Immunol 13: 679-692, 2013.

39. Krause DL and Muller N: Neuroinflammation, microglia and implications for anti-inflammatory treatment in Alzheimer's disease. Int J Alzheimers Dis 2010: pii: 732806, 2010

40. Giri S, Jatana M, Rattan R, Won JS, Singh I and Singh AK: Galactosylsphingosine (psychosine)-induced expression of cytokine-mediated inducible nitric oxide synthases via AP-1 and C/EBP: Implications for Krabbe disease. FASEB J 16: 661-672, 2002.

41. Marcheselli VL and Bazan NG: Sustained induction of prostaglandin endoperoxide synthase-2 by seizures in hippocampus. Inhibition by a platelet-activating factor antagonist. J Biol Chem 271: 24794-24799, 1996.
42. Koistinaho M and Koistinaho J: Role of p38 and p44/42 mitogenactivated protein kinases in microglia. Glia 40: 175-183, 2002.

43. Arthur JS and Ley SC: Mitogen-activated protein kinases in innate immunity. Nat Rev Immunol 13: 679-692, 2013.

44. Wang YJ, Zheng YL, Lu J, Chen GQ, Wang XH, Feng J, Ruan J, Sun X, Li CX and Sun QJ: Purple sweet potato color suppresses lipopolysaccharide-induced acute inflammatory response in mouse brain. Neurochem Int 56: 424-430, 2010.

45. Wi SM, Moon G, Kim J, Kim ST, Shim JH, Chun E and Lee KY: TAK1-ECSIT-TRAF6 complex plays a key role in the TLR4 signal to activate NF- $\kappa$ B. J Biol Chem 289: 35205-35214, 2014.

46. Hayden MS and Ghosh S: Signaling to NF-kappaB. Genes Dev 18: 2195-2224, 2004.

47. Kim KA, Lee IA, Gu W, Hyam SR and Kim DH: $\beta$-Sitosterol attenuates high-fat diet-induced intestinal inflammation in mice by inhibiting the binding of lipopolysaccharide to toll-like receptor 4 in the NF- $\kappa$ B pathway. Mol Nutr Food Res 58: 963-972, 2014. 